\title{
Patientenzufriedenheit nach strukturierter Aufklärung über invasive Eingriffe
}

\author{
B. Qarolli ${ }^{a}$, M. Schneider ${ }^{a}$, Z. Stanga ${ }^{b}$, P. Schlup ${ }^{a}$
}

\section{Zusammenfassung}

Die Einwilligung des Patienten* zu einem medizinischen Eingriff ist nur wirkungsvoll, wenn diesem eine ordnungsgemässe Aufklärung vorangegangen ist. Wir haben Patienten vor jedem medizinischen Eingriff mündlich informiert und gaben ein interventionsbezogenes Aufklärungsblatt ab. Im Anschluss an die Intervention wurde mittels Fragebogen über das Aufklärungsgespräch, den Verlauf, auffällige Komplikationen und Belastung während des Eingriffes nachgefragt. Es wurden 272 Patienten erfasst: 96\% der Patienten fühlten sich ausreichend aufgeklärt; 96\% waren mit der Zeit, welche zur Aufklärung bestand, zufrieden. Bei 92\% der Patienten verlief der Eingriff wie für sie erwartet ab. Für 94\% der Patienten verlief der Eingriff komplikationslos und 96\% waren mit dem ganzen Ablauf zufrieden. Die Resultate zeigen eine optimale Akzeptanz der schriftlichen und mündlichen Aufklärung. Mit diesen Ergebnissen können wir aus medizinischer und rechtlicher Sicht belegen, wie wichtig die Informationen über den Eingriff und die anstehende Behandlung, einschliesslich deren Wirkung und relevante Nebenwirkungen, vor dem Ereignis sind.

\section{Einleitung}

Invasive Eingriffe lösen häufig Angst und Ungewissheit aus und können mit Schmerzen und Komplikationen verbunden sein [1-3]. Gemäss eines Bundesgerichtsentscheides haben die Patienten das Recht, umfassend und wahrheitsgemäss über einen invasiven Eingriff informiert zu werden, ihn abzulehnen oder ihm zuzustimmen $[4,5]$. Die Einwilligung des Patienten zu einem medizinischen Eingriff ist nur wirkungsvoll, wenn diesem eine ordnungsgemässe Aufklärung vorangegangen ist. Nur so kann der Patient von seinem Selbstbestimmungsrecht Gebrauch machen. Durch die ärztliche Aufklärung soll dem Patienten das Verständnis vermittelt werden. Die Zustimmung des Patienten ist natürlich vor Durchführung der Behandlung abzugeben. Deshalb hat auch das Aufklärungsgespräch vorher zu erfolgen. Der Patient soll seine Entscheidung selbstbestimmt und ohne Zeitdruck treffen können [6]. Wie lange eine ausreichende Bedenkzeit ist, richtet sich wieder nach jedem Einzelfall, wie auch nach deren Dringlichkeit. Von der ärztlichen Seite wird das Informationsbedürfnis der Patienten eher unterschätzt. Potentielle Nebenwirkungen und Komplikationen werden mit ihnen oft zu wenig eingehend diskutiert [7, 8].

Zur Erhebung der Patientenzufriedenheit über die ärztliche Aufklärung von medizinischen invasiven Eingriffen führten wir diese Befragung durch. Im Rahmen eines Konzeptes zur Verbesserung der Patientensicherheit und somit zur Qualitätssicherung auf unserer Medizinischen Abteilung wollten wir wissen, wie weit die Patienten mit der ärztlichen Information über invasive Eingriffe zufrieden sind und wie stark die verschiedenen Interventionen zu Unzufriedenheit, Belastung und Komplikationen nach erweiterter Aufklärung führen kann.

\section{Methoden}

Das Kollektiv umfasste die ambulanten und stationären Patienten der medizinischen Abteilung des Spitals Grenchen, die mit einem einheitlich gestalteten Aufklärungsprotokoll über den bevorstehenden medizinischen invasiven Eingriff aufgeklärt wurden. Dabei standen die Zufriedenheit der Patienten über den Umfang, den Inhalt, die Verständlichkeit der Aufklärung und das persönliche Befinden nach dem Gespräch im Vordergrund. Ausgeschlossen wurden Patienten, die den Fragebogen nicht ohne fremde Hilfe ausfüllen konnten oder bei sprachlichen Verständigungsproblemen. Die Erhebung wurde von Mai 2001 bis Januar 2004 durchgeführt.

Vor jedem Eingriff wurden die Patienten vom zuständigen Arzt mündlich informiert und erhielten im Anschluss an das Gespräch ein interventionsbezogenes Aufklärungsblatt. Nach einem Intervall für die Lektüre des Informationsblattes wurden die Patienten vom zuständigen Arzt noch einmal aufgesucht. Wenn die Patienten die Information verstanden und mit dem Eingriff einverstanden waren, konnten sie die entspre- 
chende Erklärung unterzeichnen. Dieses Dokument wurde jeweils in der Krankengeschichte abgelegt. Mit einem Fragebogen wurden die Patienten im Anschluss an die Intervention über das Aufklärungsgespräch, den Verlauf, allfällige Komplikationen und Belastungen während des Eingriffes befragt.

Der Fragebogen bestand aus den acht folgenden Fragen:

1. Fühlten Sie sich über den Sinn des Eingriffes ausreichend aufgeklärt und über mögliche Komplikationen ausreichend informiert?

2. Stand für das Aufklärungsgespräch genügend Zeit zur Verfügung und konnten Sie Fragen stellen?

3. War das Gespräch zu ausführlich und wurden Sie dadurch verunsichert?

4. Ist der Eingriff so abgelaufen, wie Sie sich das aufgrund vorhergehender Information vorgestellt haben?

5. Wenn ein Eingriff nicht so abgelaufen ist, wie Sie sich das vorgestellt haben: Was wurde Ihnen nach Ihrer Einschätzung nicht genügend klar dargelegt?

6. Verlief der Eingriff aus Ihrer Sicht komplikationslos?

7. Sind Sie mit dem ganzen Ablauf bei diesem Eingriff, d.h. mit der Information, der Durchführung und der Nachbetreuung, zufrieden?

8. Zeichnen Sie auf der folgenden Skala (visualanalog) auf, wie stark belastend Sie den Eingriff empfunden haben.

Tabelle 1

Anzahl Untersuchungen bei 272 Patienten.

\begin{tabular}{lr} 
Untersuchungen & $\mathbf{n}$ \\
\hline Gastroskopie & 144 \\
\hline Kolonoskopie & 19 \\
\hline Pleurapunktion & 18 \\
\hline Transösophageale Echokardiographie & 16 \\
\hline Lumbalpunktion & 15 \\
\hline Rektumsigmoidoskopie & 15 \\
\hline Bronchoskopie & 11 \\
\hline Knochenmarkpunktion & 8 \\
\hline Elektrokardiokonversion & 7 \\
\hline Aszitespunktion & 6 \\
\hline ERCP & 5 \\
\hline Leberbiopsie & 2 \\
\hline PEG-Anlage & 1 \\
\hline Rektumbiopsie & 2 \\
\hline PTCA & 1 \\
\hline Holzknecht & 1 \\
\hline Magenspülung & 1 \\
\hline
\end{tabular}

Als Abgabezeitpunkt für den Fragebogen wurde der letzte oder der vorletzte Hospitalisationstag gewählt. Die Teilname an der Umfrage war freiwillig. Es bestand die Rückzugs- oder Verweigerungsmöglichkeit ohne jegliche persönliche oder medizinische Nachteile bei der weiteren Betreuung.

\section{Resultate}

Es wurden insgesamt 272 Patienten erfasst. Das Kollektiv setzte sich aus 44\% Frauen und 56\% Männer zusammen. Die Befragung wurde zu Beginn der Evaluation durch den zuständigen Stationsarzt durchgeführt $(\mathrm{n}=179)$, später durch die Teammitglieder des medizinischen Sekretariats $(n=93)$, welche weder bei der Information noch beim Eingriff involviert waren und mit dem Patienten keinen Kontakt hatten.

In der Tabelle 1 sind gesamthaft die erfassten invasiven Untersuchungen aufgelistet.

1. Frage: Fühlten Sie sich über den Sinn des Eingriffes ausreichend aufgeklärt und über mögliche Komplikationen ausreichend informiert?

$96 \%(\mathrm{n}=261) \quad$ Ja

$3 \%(\mathrm{n}=8) \quad$ Nein

$1 \%(\mathrm{n}=3) \quad$ keine Antwort

2. Frage: Stand für das Aufklärungsgespräch genügend Zeit zur Verfügung und konnten Sie Fragen stellen?

$96 \%(\mathrm{n}=261) \quad$ Ja

$3 \%(\mathrm{n}=8) \quad$ Nein

$1 \%(\mathrm{n}=3) \quad$ keine Antwort

3. Frage: War das Gespräch zu ausführlich und wurden Sie dadurch verunsichert?

$$
\begin{array}{cl}
9 \%(\mathrm{n}=24) & \text { Ja } \\
90 \%(\mathrm{n}=245) & \text { Nein } \\
1 \%(\mathrm{n}=3) & \text { keine Antwort }
\end{array}
$$

4. Frage: Ist der Eingriff so abgelaufen, wie Sie sich das aufgrund der vorhergehenden Information vorgestellt haben?

$$
\begin{array}{cl}
92 \%(\mathrm{n}=251) & \text { Ja } \\
6 \%(\mathrm{n}=16) & \text { Nein } \\
2 \%(\mathrm{n}=5) & \text { keine Antwort }
\end{array}
$$

5. Frage: Wenn der Eingriff nicht so abgelaufen ist, wie Sie sich das aufgrund der vorhergehenden Informationen vorgestellt haben: Was wurde Ihnen nach Ihrer Einschätzung nicht genügend klar dargelegt?

92\% $(\mathrm{n}=251)$ die Patienten waren mit dem Ablauf des Eingriffes zufrieden 
$6 \%(\mathrm{n}=16) \quad$ wurden nach dem Eingriff durch Husten und Würgen überrascht

$2 \%(n=5)$ die Patienten waren unzufrieden, ohne einen Grund zu nennen

6. Frage: Verlief der Eingriff aus Ihrer Sicht komplikationslos?

$94 \%(\mathrm{n}=256) \quad$ Ja

$4 \%(\mathrm{n}=11) \quad$ die Patienten hatten Komplikationen wie: Schmerzen, Erbrechen, Husten und Würgen. Keiner der befragten Patienten erlitt eine lebensbedrohliche Komplikation

$2 \%(\mathrm{n}=5) \quad$ keine Antwort

\section{Frage: Sind Sie mit dem ganzen Ablauf bei} diesem Eingriff, d.h. mit der Information, der Durchführung und der Nachbetreuung, zufrieden? $96 \%(\mathrm{n}=262) \quad$ Ja

$2 \%(\mathrm{n}=5) \quad$ Nein

$2 \%(\mathrm{n}=5) \quad$ keine Antwort

\section{Abbildung 1}

Belastung des Eingriffes mittels Visuell-analog-Skala.

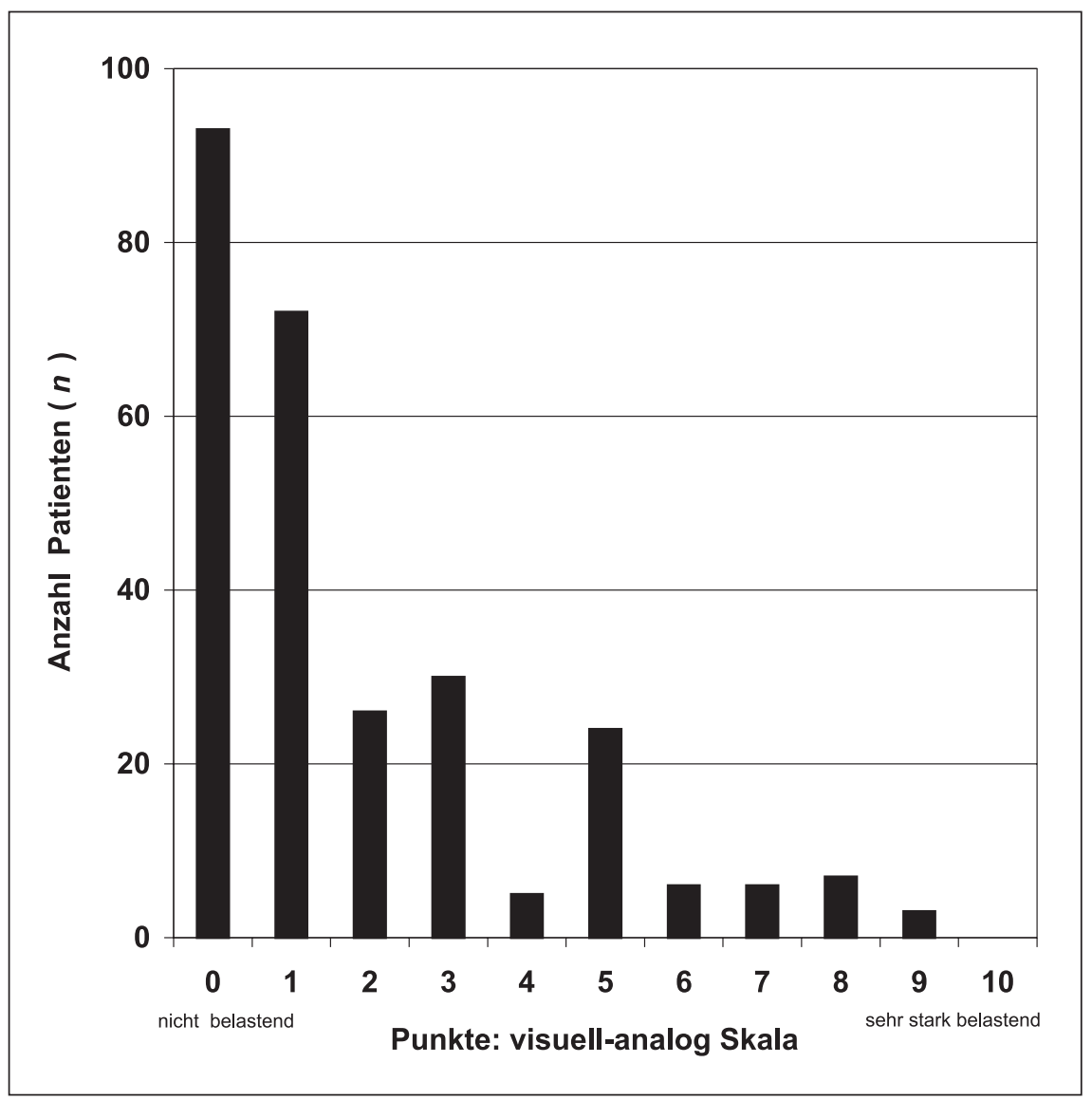

\section{Frage: Zeichnen Sie auf der folgenden} Visuell-analog-Skala von 0 (nicht belastend) bis 10 (sehr stark belastend) auf, wie stark belastend Sie den Eingriff empfunden haben? Nur 6\% der Patienten $(\mathrm{n}=16)$ gaben eine Belastung $>6$ Punkte (mittlere Belastung) bei Eingriffen nach obiger Skala an. 94\% der Patienten ( $n=256)$ gaben zu Protokoll, bei der Durchführung des Eingriffes eine Belastungsskala $<6$ Punkte (leichtere Belastung) gehabt zu haben. Interessant ist die Tatsache, dass $42 \%(\mathrm{n}=115)$ der Patienten fast keine Belastung für die Eingriffe empfunden haben.

Untersuchungen, welche mit mehr Belastung verbunden waren: vier Bronchoskopien, drei Gastroskopien, drei Koloskopien, transösophageale Echokardiographien und je eine Knochenmark-, Lumbal- und Pleurapunktion (Abb. 1).

\section{Diskussion}

Die meisten Patienten wünschen eine umfassende Aufklärung über die vorgesehene Behandlung [8]. Unsere Resultate zeigen eine gute Akzeptanz sowohl der mündlichen als auch der schriftlichen Aufklärung und eine hohe Patientenzufriedenheit mit unserem Kommunikationsund Informationskonzept. In der Literatur ist durch verschiedene andere Untersuchungen gut belegt, dass Patienten ein Aufklärungsgespräch, sogar klare und schriftliche Information positiv bewerten [9-13]. Trotzdem haben uns die sehr positiven Resultate der Umfrage erstaunt; kritisch ist in diesem Zusammenhang anzumerken, dass die Patientenbefragung zu Beginn der Studie durch den gleichen Arzt durchgeführt wurde, der vorher schon die Patienteninformation und häufig auch den Eingriff selbst vorgenommen hat. Es war deshalb nicht auszuschliessen, dass der Patient bei der abschliessenden Befragung bis zu einem gewissen Grad befangen war und sich deshalb eher positiver geäussert hat. Wie stark dieser Umstand die Resultate beeinflusst hat, blieb anfangs unklar. Aus diesem Grund führten wir die Studie weiter mit der Befragung durch neutrale Personen. Bei den letzten, insgesamt 93 Patienten wurde die abschliessende Evaluation nun ausschliesslich durch eine Praxisassistentin durchgeführt. Diese war den Patienten unbekannt. Bei der Auswertung fanden wir bei allen acht Fragen keinen signifikanten Unterschied zwischen der Evaluation beider Befragungen. Mit dieser Umfrage konnten wir nochmals beweisen, wie wichtig es aus medizinischer und 
rechtlicher Sicht ist, Informationen auf eine einfache Art und Weise über einen Eingriff und die anstehende Behandlung, einschliesslich deren Wirkung und relevante Nebenwirkungen, vor der Untersuchung abzugeben. Das Aufklärungsgespräch spielte eine prioritäre Rolle während der ganzen Befragung. Der Umfang an Aufklärung musste den Informationswünschen, der Verständnisfähigkeit und Belastbarkeit des einzelnen Patienten gerecht werden. Die genutzten Aufklärungsprotokolle waren eine gute Ergänzung für das Gespräch wie auch ein gutes Hilfsmittel für die Dokumentation des Aufklärungsgespräches.

\section{Danksagung}

Diese Aufgabe zu bewältigen war nur möglich durch das aussergewöhnliche Engagement und das ausgesprochen reibungslose und kooperative Zusammenspiel aller Beteiligten auf der medizinischen Abteilung des Spitals Grenchen; dafür danken wir insbesondere den Teammitgliedern des medizinischen Sekretariats. Schliesslich ein grosses Dankeschön an alle, die als Befragte an der Studie teilgenommen und ebenso bereitwillig wie interessiert und freimütig Auskunft gegeben haben.

\section{Literatur}

1 Leape LL, Brennan TA, Laird N, Lawthers AG, Localio AR, Barnes BA, et al. The nature of adverse events in hospitalized patients. N Engl J Med 1991;324:377-84.

2 Brennan TA, Leape LL, Laird NM, Hebert L, Localio AR, Léawthers AG, et al. Incidence of adverse events and negligence in hospitalized patients. Results of the Harvard Medical Practice Study I. N Engl J Med 1991;324:370-6.
3 Stäubli M, Suter J. Die Komplikationenliste der Schweizerischen Gesellschaft für Innere Medizin. Schweiz Ärztezeitung 2004;85(21):1109-16.

4 Bundesgerichtsentscheide (BGE) 113 I b 426 E. 6; 108 II 61 E. 2; 117 I b E. 3.

5 Bertschinger P. Informed Consent. Einverständniserklärung vor invasiven gastroenterologischen Untersuchungen in der Schweiz. Schweiz Ärztezeitung 2002;83(13):603-5.

6 Roggo A. Aufklärung des Patienten. Eine ärztliche Informationspflicht. Bern: Stämpfli; 2002.

7 Doust JA, Morgan TN, Weller BJ, Yuill BJ. Patient desire for information before a total hip replacement operation. Med J Aust 1989;151:201-3.

8 Wallace LM. Informed consent to elective surgery: the "therapeutic" value? Soc Sci Med 1986; 22:29-33.

9 Kessler M, Faisst K, Kessler W. Patientenzufriedenheit nach Operationsaufklärung mit dem Aufklärungsprotokoll der Schweizerischen Gesellschaft für Chirurgie (SGC). Schweiz Ärztezeitung 2000;81(34):1852-6.

10 Shepherd HA, Bowman D, Hancock B, Anglin J, Hewett D. Postal consent for upper gastrointestinal endoscopy. Gut 2000;46:37-9.

11 Askew G, Pearson KW, Cryer D. Consent: can we educate patients? J R Coll Surg Edinb 1990; 35:308-10.

12 Edwards MH. Satisfying patients' needs for surgical information. Br J Surg 1990;77:463-5.

13 Armstrong AP, Cole AA, Page RE. Informed consent: are we doing enough. Br J Plast Surg 1997;50:637-40.

14 Roggo A. Roadmap Aufklärung von Patienten. In: Fellmann W, Poledna T (Hrsg.). Die Haftung des Arztes und des Spitals, Fragen und Entwicklungen im Recht der Arzt- und Spitalhaftung. Zürich: Forum Gesundheitsrecht / droit de la santé; 2003. S. 73-109.

15 Bundesgerichtsentscheide (BGE) 66 II 34, 36. 